\title{
Vitamins C and K3: A Powerful Redox System for Sensitizing Leukemia Lymphocytes to Everolimus and Barasertib
}

\author{
DONIKA IVANOVA $^{1}$, ZHIVKO ZHELEV ${ }^{1,2}$, DESSISLAVA LAZAROVA ${ }^{3}$, \\ PLAMEN GETSOV $^{4}$, RUMIANA BAKALOVA ${ }^{3,5,6}$ and ICHIO AOKI ${ }^{5,6}$ \\ ${ }^{1}$ Medical Faculty, Trakia University, Stara Zagora, Bulgaria; \\ ${ }^{2}$ Institute of Biophysics and Biomedical Engineering, Bulgarian Academy of Sciences, Sofia, Bulgaria; \\ ${ }^{3}$ Medical Faculty, Sofia University, Sofia, Bulgaria; \\ ${ }^{4}$ University Hospital "Tsaritsha Yoanna-ISUL", Sofia, Bulgaria; \\ ${ }^{5}$ Department of Molecular Imaging and Theranostics, and \\ ${ }^{6}$ Quantum-state Controlled MRI, National Institute of Radiological Sciences, \\ National Institute for Quantum and Radiological Science and Technology, Chiba, Japan
}

\begin{abstract}
Background/Aim: Recent studies provided convincing evidence for the anticancer activity of combined application of vitamin $C$ and pro-vitamin $K 3$ (menadione). The molecular pathways underlying this process are still not well established. The present study aimed to investigate the effect of the combination of vitamin $C$ plus pro-vitamin $K 3$ on the redox status of leukemia and normal lymphocytes, as well as their sensitizing effect for a variety of anticancer drugs. Materials and Methods: Cytotoxicity of the substances was analyzed by trypan blue staining and automated counting of live and dead cells. Apoptosis was analyzed by fluorescein isothiocyanate-annexin $V$ test. Oxidative stress was evaluated by the intracellular levels of reactive oxygen and nitrogen species and protein-carbonyl products. Results: Combined administration of $300 \mu \mathrm{M}$ vitamin C plus $3 \mu \mathrm{M}$ pro-vitamin $\mathrm{K} 3$ reduced the viability of leukemia lymphocytes by $\sim 20 \%$, but did not influence the viability of normal lymphocytes. All combinations of anticancer drug plus vitamins $C$ and $K 3$ were characterized by synergistic cytotoxicity towards Jurkat cells, compared to cells treated with drug alone for $24 \mathrm{~h}$. In the case of barasertib and everolimus, this synergistic cytotoxicity increased within 72 hours. It was accompanied by strong induction of apoptosis, but a reduction of level of hydroperoxides and
\end{abstract}

Correspondence to: Rumiana Bakalova, Ph.D., D.Sci., Department of Molecular Imaging and Theranostics, National Institutes for Quantum and Radiological Science and Technology (QST/NIRS), 4-9-1 Anagawa, Inage-ku, Chiba 263-8555, Japan. E-mail: bakalova.rumiana@qst.go.jp

Key Words: Vitamin C, pro-vitamin K3, apoptosis, oxidative stress, Jurkat, leukemia lymphocytes. moderately increased protein-carbonyl products in leukemia cells. Conclusion: Leukemia lymphocytes were more sensitive to combined administration of anticancer drug (everolimus or barasertib) plus vitamins $C$ and $K 3$, compared to normal lymphocytes. The combination of vitamin $C$ plus $K 3$ seems to be a powerful redox system that could specifically influence redox homeostasis of leukemia cells and sensitize them to conventional chemotherapy.

It is widely accepted that the efficiency of conventional chemotherapeutics, as well as their harmful side-effects, are due to disruption of redox homeostasis and induction of oxidative stress in normal cells and tissues $(1,2)$. In this case, natural and synthetic antioxidants are used as supplements to control the process. Many experimental studies have shown a decrease of reactive oxygen species (ROS) after administration of anticancer drugs combined with antioxidants (3-5). However, other studies have reported that many natural compounds recognized as antioxidants can also exhibit pro-oxidant effects, depending on environmental conditions (6-8). It is assumed that these natural compounds do not affect the efficiency of chemotherapy, but induce cancer progression due to induction of drug resistance. In our previous studies, we found that certain redox modulators (e.g. melatonin, 2-deoxy-D-glucose, 6-aminonicotinamide, and docosahexaenoic acid) sensitize leukemia lymphocytes to anticancer drugs (9-12). Some combinations (redox modulator plus anticancer drug) are characterized by synergistic cytotoxicity towards leukemia cells, but without elevated ROS production. In addition, these redox modulators did not significantly affect cell viability or the level of ROS and did not induce apoptosis of normal lymphocytes when combined with new-generation anticancer drugs (e.g. everolimus and barasertib) (9-12). 
Some of the most interesting natural compounds are vitamins $\mathrm{C}$ and $\mathrm{K}$, and especially their combination. There is convincing evidence for cell-cycle arrest and cell death after combined administration of vitamin $\mathrm{C}$ and pro-vitamin K3 (menadione) to different cancer cell lines (13-16). The molecular pathways underlying this process are still not well established. It is assumed that the interaction between vitamin $\mathrm{C}$ and $\mathrm{K} 3$ leads to generation of superoxide radicals, followed by production of hydrogen peroxide and induction of oxidative stress in cancer cells (13-16). Zhang et al. also showed that cancer cells derived from oral squamous carcinoma (HSC-2, HSC-3) and promyelocytic leukemia (HL-60) are more sensitive to combined administration of vitamin $\mathrm{C}$ and $\mathrm{K} 3$, in comparison with normal cells (17). Their study supports our assumption that the changes in redox homeostasis of cancer cells by targeting specific biochemical/metabolic pathways involved in oncogenesis and cancer progression could be used as a new therapeutic strategy (18).

The present study aimed to investigate the effect of vitamin $\mathrm{C}$ and $\mathrm{K} 3$ on the redox status of leukemia and normal lymphocytes, as well as their sensitizing effect to variety of anticancer drugs - four conventional and 11 newgeneration drugs. We also discuss the molecular mechanisms of action of vitamin $\mathrm{C}$ and $\mathrm{K} 3$ in combination, based on regulation of metabolic processes, which are vital for cancer cells, but not for normal cells.

\section{Materials and Methods}

Cells and treatment protocol. The experiments were performed on leukemia lymphocytes (Jurkat), derived from patients with acute lymphoblastic leukemia, as well as on normal lymphocytes isolated from healthy blood donors using Lymphosepar-I (ImmuneBiological Laboratories Co., Fujioka, Japan). The cells were cultured in RPMI-1640 medium (Sigma-Aldrich, Steinheim, Germany), supplemented with $10 \%$ heat-inactivated fetal bovine serum (Gibco, Auckland, New Zealand) and antibiotics $(100 \mathrm{U} / \mathrm{ml}$ penicillin and $100 \mu \mathrm{g} / \mathrm{ml}$ streptomycin) (Gibco) in a humidified atmosphere at $37^{\circ} \mathrm{C}$ with $5 \% \mathrm{CO}_{2}$. All cells were collected by centrifugation $(1,000 \times g$ for $10 \mathrm{~min})$ and replaced in a fresh medium without antibiotics before treatment with anticancer drugs.

The drugs were dissolved in dimethyl sulfoxide (DMSO; suitable for cell cultures; Sigma-Aldrich) or phosphate-buffered saline (PBS; $10 \mathrm{mM}, \mathrm{pH}$ 7.4). The final concentration of DMSO in the cell suspension did not exceed $1 \%$. At this concentration, DMSO did not influence cell viability.

The cells $\left(1 \times 10^{6}\right.$ cells $\left./ \mathrm{ml}\right)$ were incubated with vitamin $\mathrm{C}$ and $\mathrm{K} 3$, drug, or drug plus vitamin $\mathrm{C}$ and $\mathrm{K} 3$ at the following concentrations: $300 \mu \mathrm{M}$ vitamin $\mathrm{C}$ (Sigma-Aldrich), $3 \mu \mathrm{M}$ vitamin K3 (Sigma-Aldrich), $0.25 \mu \mathrm{M}$ palbociclib (Selleckchem, Houston, TX, USA), $0.5 \mu \mathrm{M}$ PI-103, $5 \mu \mathrm{M}$ (Selleckchem), everolimus (Selleckchem), $0.5 \mu \mathrm{M}$ lonafarnib (Selleckchem), $0.1 \mu \mathrm{M}$ ABT-737 (Selleckchem), $0.1 \mu \mathrm{M}$ doxorubicin (Sigma-Aldrich), $0.5 \mu \mathrm{M}$ bleomycin (Nippon Kayaku Co., Tokyo, Japan), $0.1 \mu \mathrm{M}$ AZD-7762 (Sigma-Aldrich), $0.01 \mu \mathrm{M}$ MLN-2238 (Selleckchem), $0.025 \mu \mathrm{M}$
MG-132 (Wako, Tokyo, Japan), $10 \mu \mathrm{M}$ lomustine (Sigma-Aldrich), $2.5 \mu \mathrm{M}$ cisplatin (Selleckchem), $0.025 \mu \mathrm{M}$ BEZ-235 (Selleckchem), $0.01 \mu \mathrm{M}$ bortezomib (Selleckchem), $0.05 \mu \mathrm{M}$ or $0.01 \mu \mathrm{M}$ barasertib (Selleckchem). Drugs were applied to the cells at these concentrations (single dose) and incubated for different time intervals in a cell incubator. At each time interval, aliquots were used for cell viability assay. The selected concentrations of drugs, as well as of combinations (vitamin C plus K3), when applied separately induced about $20 \%$ inhibition of the growth of leukemia lymphocytes.

Cell viability assay. Cell viability was analyzed using trypan blue staining and Countess ${ }^{\mathrm{TM}}$ Automated Cell Counter (Invitrogen, Eugene, OR, USA) at very precise standardization of the measurements. Three independent experiments (with two repetitive measurements) for each experiment were performed for each sample. Non-treated cells were used as controls. The data are presented as the mean $\pm \mathrm{SD}$.

Intracellular ROS assay. The amount of ROS was analyzed using OxiSelect $^{\mathrm{TM}}$ In vitro ROS/RNS Assay Kit - Green Flourescence (Cell Biolabs., Inc., San Diego, CA, USA). The method is based on the use of fluorogenic probe 2', 7'-dichlorodihydrofluorescin (DCFHDiOxyQ). In the cytosol, the probe is deacetylated to the nonfluorescent DCFH. DCFH reacts with ROS and reactive nitrogen species (RNS) (predominantly $\mathrm{H}_{2} \mathrm{O}_{2}$, $\mathrm{ROO}^{\circ}, \mathrm{NO}, \mathrm{ONOO}^{-}$) with formation of fluorescent product $2^{\prime}, 7^{\prime}$ '-dichlorodihydrofluorescein (DCF). The intensity of DCF fluorescence $\left(\lambda_{\mathrm{ex}}=480 \mathrm{~nm}, \lambda_{\mathrm{em}}=530\right.$ $\mathrm{nm}$ ) is proportional to the amount of ROS/RNS in the biological sample.

The amount of ROS/RNS was calculated by calibration curve based on DCF standard solution in PBS. The measurements were performed on a Tecan Infinite F200 PRO (Tecan Austria GmbH, Mannedorf, Austria) microplate reader.

Briefly, the cells $\left(1 \times 10^{6}\right.$ cells $\left./ \mathrm{ml}\right)$ were collected by centrifugation $(1000 \times g$ for $10 \mathrm{~min})$ and lysed using $300 \mu \mathrm{l}$ of $0.1 \%$ sodium dodecylsulfate (SDS; dissolved in PBS) within $30 \mathrm{~min}$ on ice. The lysates were adjusted to equal protein concentration (in the range $1-10 \mathrm{mg} / \mathrm{ml}$ ) using PBS. Protein concentration was analyzed by Bradford assay. Each sample was subjected to ROS/RNS assay, according to the manufacturer's instruction.

Protein-carbonyl assay. The amount of protein-carbonyl products were analyzed using OxiSelect ${ }^{\mathrm{TM}}$ Protein Carbonyl Spectrophotometric Assay Kit (Cell Biolabs, Inc.). The most common products of protein oxidation in biological samples are the protein-carbonyl derivatives of proline, arginine, lysine and threonine, which are chemically stable and serve as markers of oxidative stress. The analysis of these products is based on derivatization of the carbonyl groups with dinitrophenylhydrazine with formation of protein-hydrazone. The amount of protein-hydrazone was detected spectrophotometrically at $375 \mathrm{~nm}$.

Briefly, the cells $\left(1 \times 10^{6}\right.$ cells $\left./ \mathrm{ml}\right)$ were collected by centrifugation $(1000 \times g, 10 \mathrm{~min})$ and lysed using $300 \mu \mathrm{l}$ of $0.1 \%$ SDS (dissolved in PBS) within 30 min on ice. The lysates were adjusted to equal protein concentration (in the range $1-10 \mathrm{mg} / \mathrm{ml}$ ) using PBS. Protein concentration was analyzed by Bradford assay. Each sample was subjected to protein-carbonyl assay, according to the manufacturer's instruction. Oxidized bovine serum albumin was used as a standard. 
Apoptosis assay. Induction of apoptosis was analyzed by the expression of phosphatidylserine (PSer) on the cell surface, using fluorescein isothiocyanate (FITC)-Annexin V Apoptosis Detection Kit (BioVision, Milpitas, CA, USA).

Briefly, the cells $\left(1 \times 10^{6}\right.$ cells $\left./ \mathrm{ml}\right)$ were incubated with drug, vitamin $\mathrm{C}$ plus K3, or their combination, under the conditions mentioned above. At different time-points, the cells were collected by centrifugation $(1000 \times g$ for $10 \mathrm{~min})$, washed twice with PBS containing $2.5 \mathrm{mM}$ $\mathrm{CaCl}_{2}$ (annexin V-binding buffer) and re-suspended in the same buffer. One hundred microliters of the suspension were incubated with $5 \mu \mathrm{l}$ of FITC-annexin $\mathrm{V}$ for $10 \mathrm{~min}$ at room temperature in the dark. The cells were washed three times with annexin V-binding buffer and were finally re-suspended in $500 \mu \mathrm{l}$ of the same buffer. FITC-annexin V bound to PSer exposed on the cell surface was detected spectrofluorimetrically at $\lambda_{\mathrm{ex}}=488 \mathrm{~nm}$ and $\lambda_{\mathrm{em}}=535 \mathrm{~nm}$, using a Tecan Infinite F200 PRO (Tecan Austria GmbH) microplate reader.

\section{Results and Discussion}

Effect of the combination of vitamin $C$ and $K 3$ on the viability of leukemia and normal lymphocytes. The cells were incubated with different concentrations of vitamin C and K3, keeping the ratio at 100:1 (mol:mol). It has been shown that the anticancer effect of the combinations of both vitamins is most strongly expressed at this molar ratio (13-16). The aim of this stage of the study was to select the optimal concentrations of the combination of both vitamins, which induced $\sim 20-30 \%$ cytotoxicity in leukemia lymphocytes after 48-hour incubation. This level of cytotoxicity enables assessment of synergism, additivity or antagonism after combined application of vitamin $\mathrm{C}$ and $\mathrm{K} 3$ with anticancer drugs.

The data in Figure 1 demonstrate that combined administration of $300 \mu \mathrm{M}$ vitamin $\mathrm{C}$ plus $3 \mu \mathrm{M}$ vitamin $\mathrm{K} 3$ reduced the viability of leukemia lymphocytes by $20 \%$ (Figure 1A) but did not influence the viability of normal lymphocytes (Figure 1B). A similar effect was also detected for combined application of $400 \mu \mathrm{M}$ vitamin $\mathrm{C}$ plus $4 \mu \mathrm{M}$ pro-vitamin K3. Above these concentrations, the combination of both vitamins induced cytotoxicity not only in leukemia lymphocytes, but also in normal lymphocytes.

Effect of vitamin $C$ and $K 3$ on the cytotoxicity of anticancer drugs towards leukemia lymphocytes. The second stage of the study aimed to clarify the effect of vitamin C and K3 (300 $\mu \mathrm{M}$ vitamin $\mathrm{C}$ plus $3 \mu \mathrm{M}$ pro-vitamin $\mathrm{K} 3$ ) on the cytotoxicity of anticancer drugs towards Jurkat leukemia cells.

Cells were incubated with vitamin C plus K3 and anticancer drug - separately and in combination, for different time intervals $(24,48$ and $72 \mathrm{~h})$. The results are shown in Figure 2. The calculations are described in detail in our previous article (9). Briefly, to distinguish the synergistic cytotoxic effect from antagonistic/additive effects, we calculated the effect of each combination on cell proliferation as a percentage of the effect of the respective drug applied alone. Then we compared this effect to the effect of the combination (vitamin C plus K3),
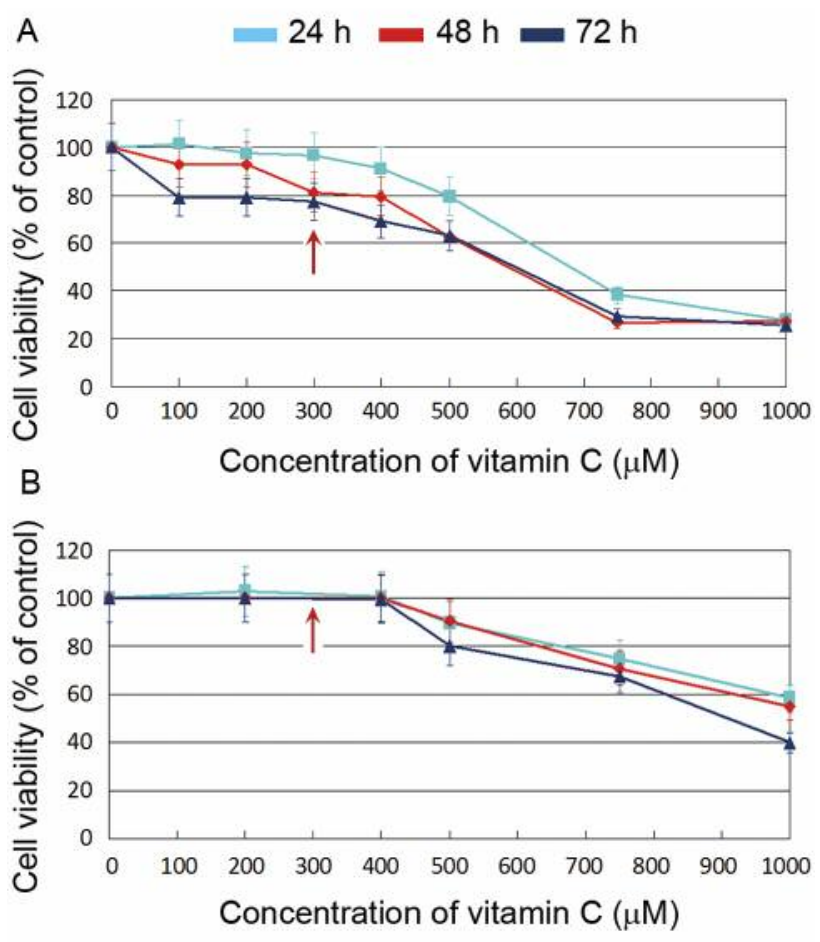

Figure 1. Effect of vitamin $C$ and $K 3$ on viability of Jurkat leukemia lymphocytes $(A)$ and normal lymphocytes $(B)$ after different incubation times. The data are the mean $\pm S D$ of six independent experiments. The arrows indicate the concentrations of vitamin $C(300 \mu M)$ and $K 3(3 \mu M)$ selected for further experiments in combination with anticancer drugs.

when applied alone. The effect of vitamin $\mathrm{C}$ plus $\mathrm{K} 3$ is indicated by the dashed line in Figure 2B. In the case of drug plus vitamin $\mathrm{C}$ and $\mathrm{K} 3$, the data located to the left of the dashed line represent synergistic cytotoxic effects, while the data located to the right of the dashed line represent antagonistic effects. All data matching the dashed line reflect additive effects. The cytotoxicity of each drug applied alone at the selected concentration ranged from $\sim 10-20 \%$ after $24-\mathrm{h}$ incubation to $\sim 20-30 \%$ after 48 - and 72-h incubation. After 24-hour incubation, all combinations (anticancer drug plus vitamin $\mathrm{C}$ and $\mathrm{K} 3$ ) were characterized by synergistic cytotoxicity towards Jurkat cells compared to cells treated with drug only (Figure 2B). However, this synergistic cytotoxicity decreased or disappeared after 48- and 72-h incubation. Only in the case of barasertib and everolimus we observed a clear enhancement of the synergistic cytotoxicity with vitamin $\mathrm{C}$ and $\mathrm{K} 3$ within $72 \mathrm{~h}$.

Effect of vitamin $C$ and $\mathrm{K} 3$ on everolimus- and barasertibinduced cytotoxicity, apoptosis, production of ROS and protein-carbonyl products in leukemia and normal lymphocytes. The third stage of our study aimed to investigate whether the strong synergistic cytotoxicity of 
A

$\square$ Vit. C\&K3 only $\square$ Drug only $\square$ Combination

B
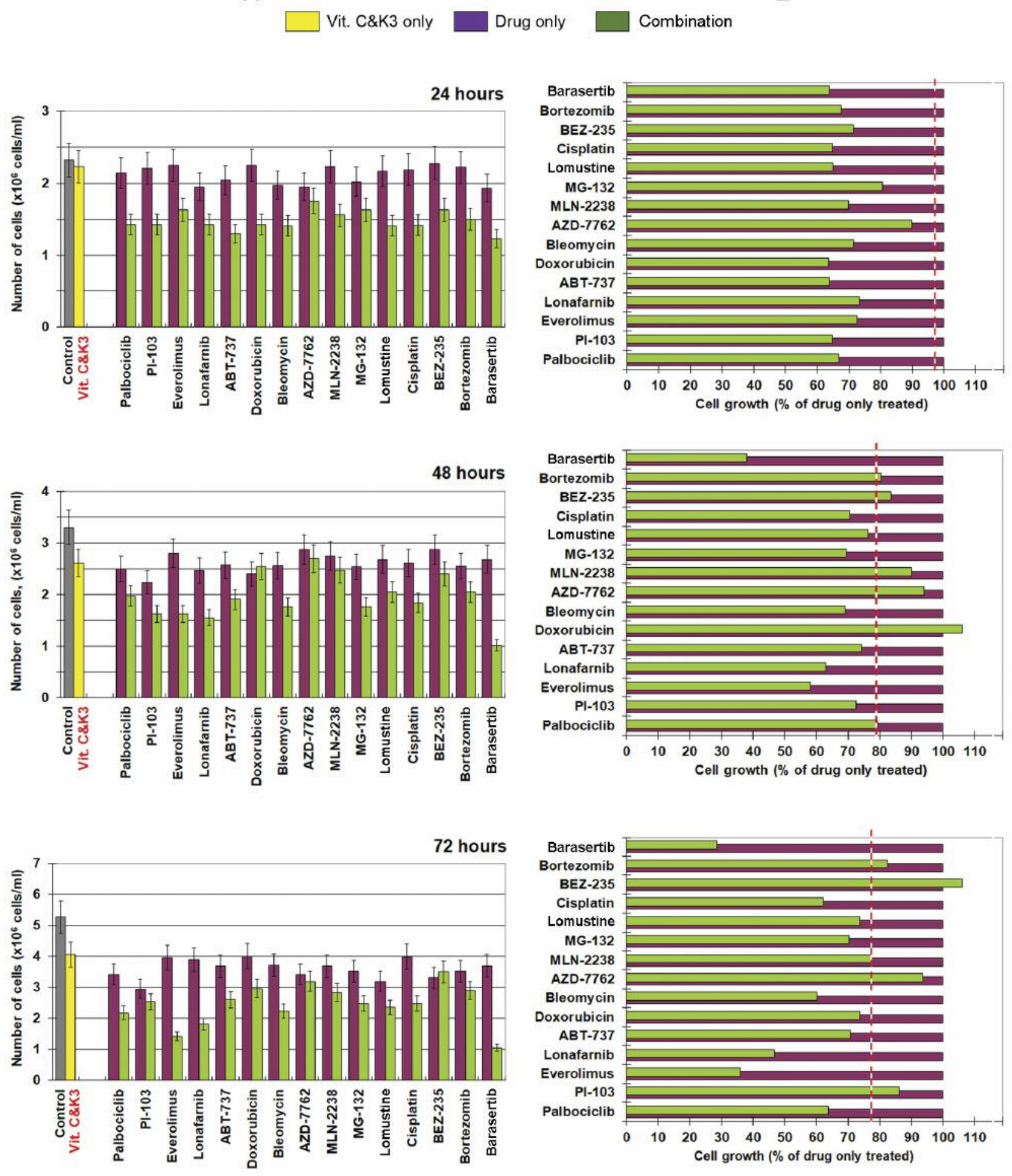

Figure 2. A: Effect of vitamin $C$ and $K 3$ (Vit. C\&K3) and anticancer drugs alone and in combination on cell proliferation of Jurkat cells after incubation for 24-, 48- and 72-h. Incubation conditions: $1 \times 10^{6}$ cells $/ \mathrm{ml}$, redox-modulators $(300 \mu M$ vitamin $C$ plus $3 \mu M$ pro-vitamin K3) with/without or drug (at concentrations given in the Materials and Methods), at $37^{\circ} \mathrm{C}$ in a humidified atmosphere. Data are the mean $\pm S D$ of six independent experiments. B: Effect of each combination (drug plus Vit. C\&K3) on cell proliferation as a percentage of the effect of drug when applied alone. The dashed line indicates the effect of Vit. $C \& K 3$ on cell proliferation activity of cancer cells as a percentage that of the control (untreated cells). *In this experiment, the concentration of barasertib was $50 \mathrm{nM}$. 
A

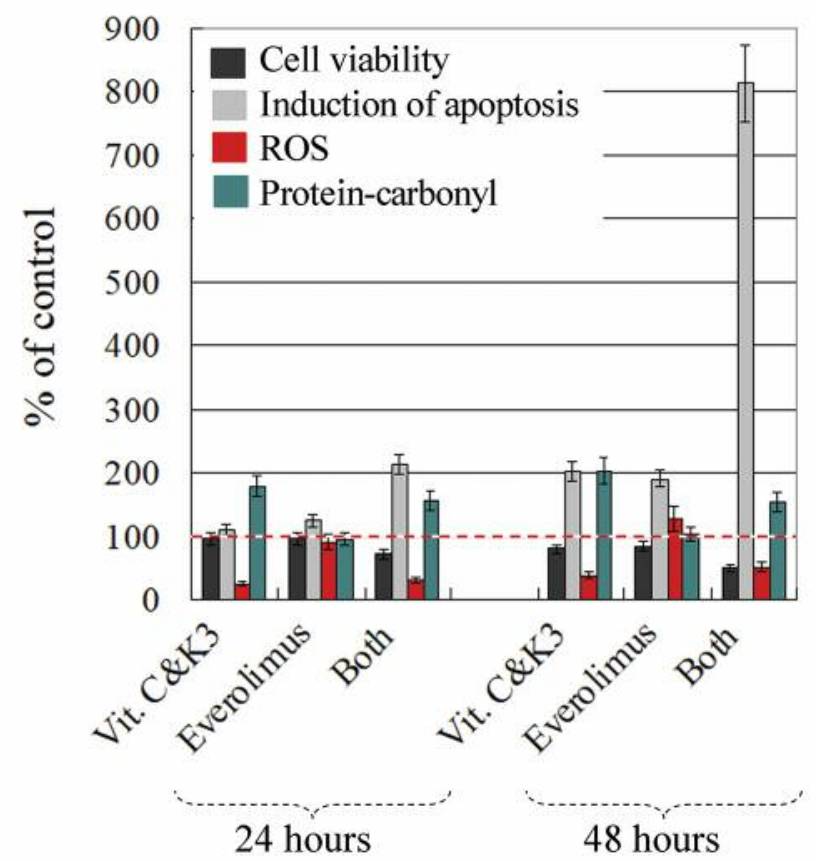

B

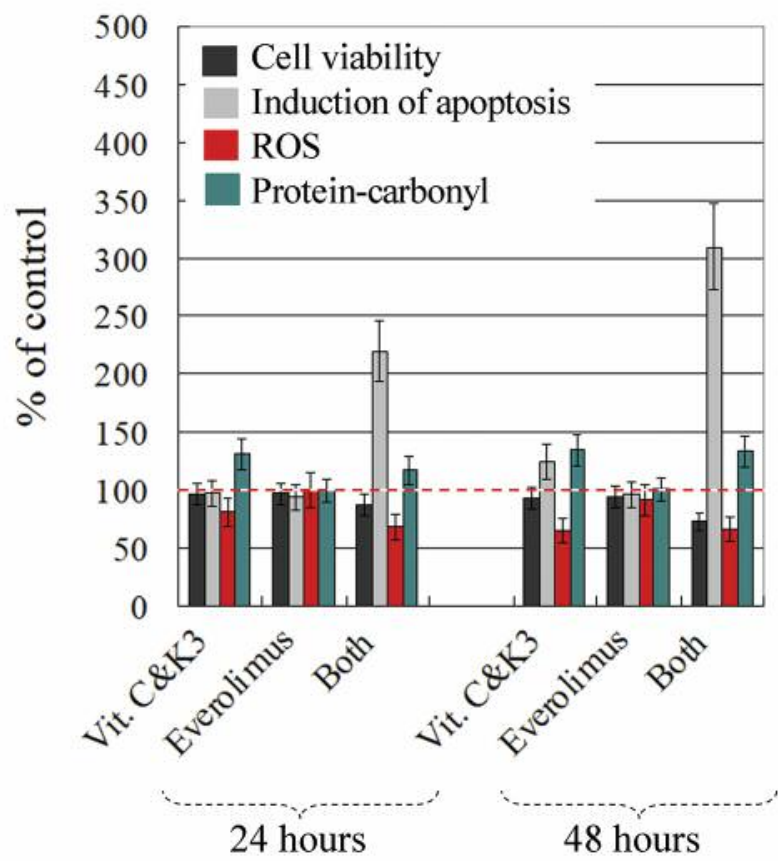

Figure 3. Effects of vitamin $C$ and $K 3$ (Vit. $C \& K 3 ; 300 \mu M$ and $1 \mu M$, respectively), everolimus $(5 \mu M)$ and their combination on cell viability, induction of apoptosis, level of reactive oxygen species (ROS) and level of protein-carbonyl products in Jurkat leukemia cells (A) and normal lymphocytes (B), after 24- and 48-hour incubation at 37oC in humidified atmosphere. The data are the mean $\pm S D$ from three independent experiments.

A

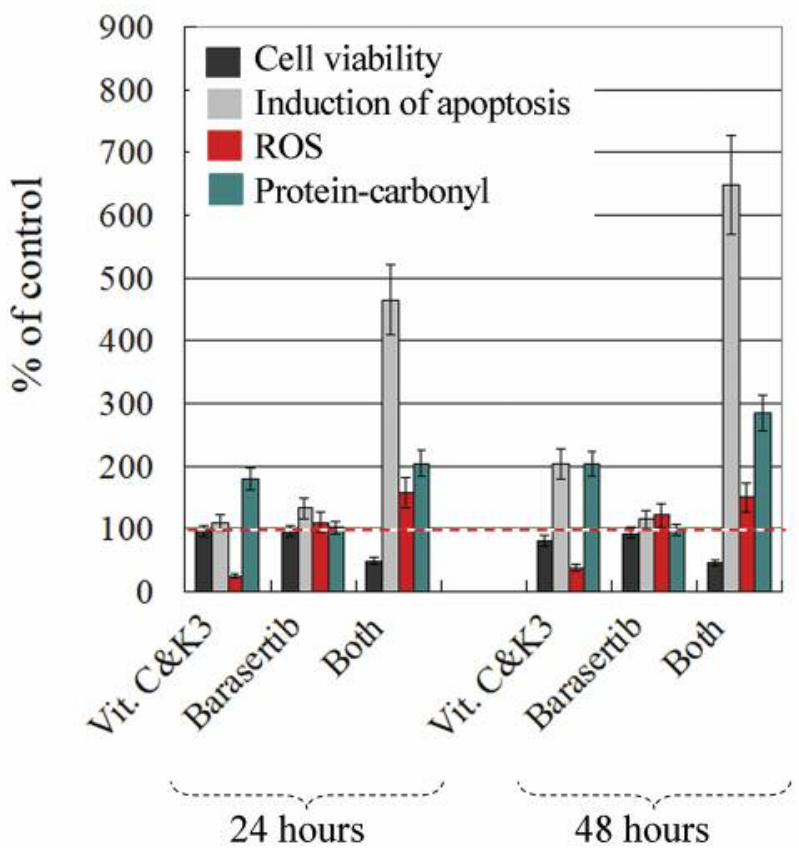

B

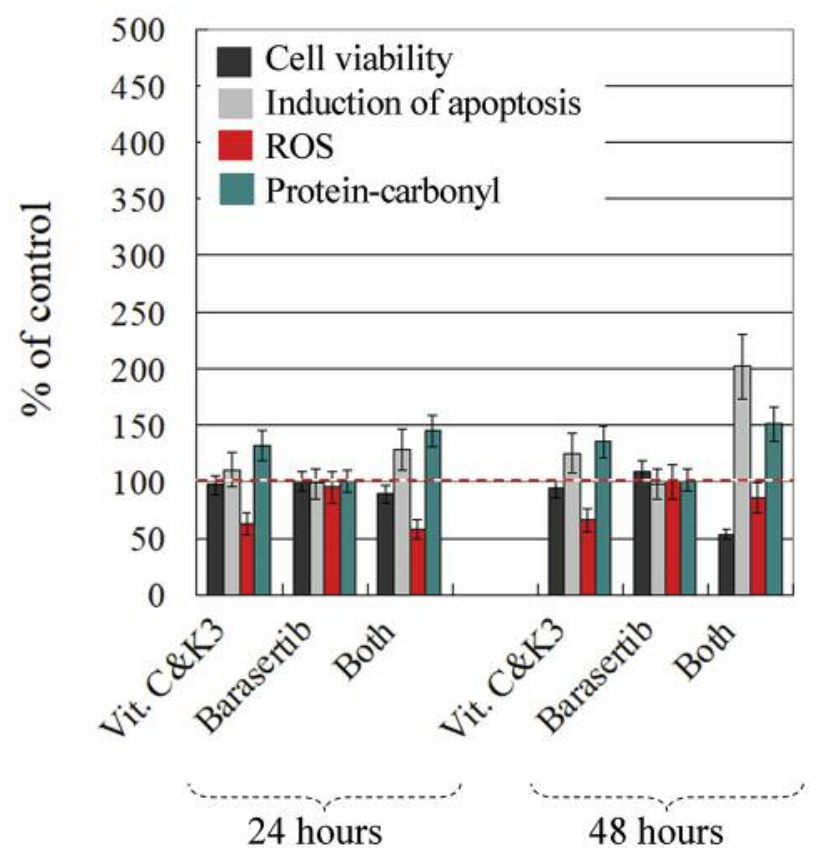

Figure 4. Effects of vitamin $C$ and $K 3$ (Vit. C\&K3; $300 \mu M$ and $1 \mu M$, respectively), barasertib (0.01 $\mu M)$ and their combination on cell viability, induction of apoptosis, level of reactive oxygen species (ROS) and level of protein-carbonyl products in Jurkat leukemia cells (A) and normal lymphocytes $(B)$, after 24-and 48-h incubation at $37^{\circ} \mathrm{C}$ in humidified atmosphere. The data are the mean $\pm S D$ from three independent experiments. 
vitamin $\mathrm{C}$ and $\mathrm{K} 3$ in combination with barasertib or everolimus towards leukemia lymphocytes was accompanied by induction of oxidative stress. To estimate the possible side-effects, we analyzed the effects of these combinations on the viability and redox status of normal lymphocytes.

Everolimus is an inhibitor of the mammalian target of rapamycin (mTOR) pathway, which has a fundamental role in the regulation of cell viability, translational initiation, and cell-cycle progression. Recent studies have reported that everolimus has anticancer effects (19-22). The drug sensitizes cancer cells to other anticancer drugs, as well as preventing the development of multidrug resistance through altering the balance between pro-apoptotic and anti-apoptotic factors $(20,21)$.

Barasertib belongs to a new class of pyrazoloquinazolines, selective inhibitors of aurora B kinase (23). Currently, the aurora kinases are the subject of considerable interest as targets for the development of new anticancer agents. Little is known about the molecular mechanisms of action of barasertib, except that it inhibits aurora B kinase, provokes cell-cycle arrest and apoptosis, as well as enhancing the response to chemotherapy (24).

Some researchers have shown that the anticancer effect of everolimus and barasertib was not mediated by increased production of ROS (25), but others suggest the involvement of ROS in this process (26-28). In our previous studies, we established that everolimus and barasertib in combination with redox modulators induced apoptosis of leukemia lymphocytes via ROS-independent (in the case of 2-deoxyD-glucose, 6-aminonicotinamide, and melatonin) or ROSdependent mechanisms (in the case of docosahexaenoic acid) (9-12).

In this study, everolimus and barasertib were used at very low concentrations that had no significant effect on cell viability when the drug was applied alone to both cell types.

The data in Figure 3 show that everolimus, at $5 \mu \mathrm{M}$ exhibited a slight cytotoxic effect ( $10 \%$ after 48 -hour incubation) towards leukemia lymphocytes, but not towards normal lymphocytes. In Jurkat cells, the cytotoxicity of everolimus applied alone was accompanied by induction of apoptosis, but only a relatively slight (insignificant) increase of the level of ROS, and without change in the level of protein-carbonyl products (Figure 3A). Vitamin C and K3 increased the cytotoxicity of everolimus by up to $30 \%$ after 24-hour and $250 \%$ after 48 -hour incubation with leukemia lymphocytes (Figure 3A). In Jurkat cells, the combination exhibited a very strong induction of apoptosis $(\sim 8$-fold that of the control after 48-hour incubation). This effect was accompanied by a decrease of ROS even below the level of the control (Figure 3A). However, the combination of everolimus with vitamin $\mathrm{C}$ and $\mathrm{K} 3$ also affected the viability of normal lymphocytes - by up to $15 \%$ after 24 -hour and $\sim 25 \%$ after 48-hour incubation (Figure 3B). This effect was accompanied by induction of apoptosis ( 2- to 3-fold that of the control), reduction of ROS ( $30-35 \%)$, but enhancement of protein-carbonyl products $(\sim 30-50 \%)$ (Figure $3 \mathrm{~B})$.

Our data indicate that the high levels of ROS (e.g. hydroperoxides analyzed by DCF test) are not obligatory for induction of apoptosis in leukemia lymphocytes treated with everolimus plus vitamin C and K3. Nevertheless, an elevated level of protein-carbonyl products indicates induction of oxidative stress in both cell types. This can explain, at least partially, the side-effect of this combination on normal lymphocytes. It seems that vitamin $\mathrm{C}$ and $\mathrm{K} 3$ are mainly responsible for altering the redox homeostasis of normal lymphocytes: protein-carbonyl products increased even when the combination of both vitamins was applied in the absence of everolimus.

On the other hand, recent studies have reported that everolimus sensitizes malignant pleural mesothelioma and osteosarcoma cells to sorafenib by triggering ROS-induced apoptosis mediated through adenosine monophosphateactivated protein kinase $(26,27)$. It was also found that the level of oxidized glutathione increased and the levels of reduced glutathione and NADPH decreased in mice with ischemia-reperfusion injury treated with everolimus (29). Dando et al. have shown that everolimus induces nuclear translocation of the cytosolic glycolytic enzyme glyceraldehyde 3-phosphate dehydrogenase (GAPDH) in pancreatic adenocarcinoma cells (30). GAPDH is a redoxsensitive protein and its activity is regulated by reducers and oxidizers. This mechanism is being discussed as a reason for synergistic cytotoxicity between everolimus and genipin (an inhibitor of the mitochondrial uncoupling protein 2).

In the case of barasertib, the cells were treated with a very low concentration of the drug $(10 \mathrm{nM})$. At this concentration, barasertib did not affect the viability of either cell type during 24- and 48-hour incubation (Figure 4). However, the combination of barasertib plus vitamin $\mathrm{C}$ and $\mathrm{K} 3$ induced a strong cytotoxic effect on leukemia cells ( 45-50\%), which was accompanied by a strong induction of apoptosis (6-fold that of the control after $48 \mathrm{~h}$ ) and a relatively moderate enhancement of ROS and protein-carbonyl levels, as detected after 24- and 48-hour incubation (Figure 4A). Unfortunately, the combination of barasertib plus vitamin $\mathrm{C}$ and $\mathrm{K} 3$ exhibited cytotoxicity towards normal lymphocytes ( $\sim 10 \%$ after 24 -hour and $\sim 45 \%$ after 48 -h incubation) (Figure 4B). This cytotoxic effect was accompanied by induction of apoptosis and enhancement of protein-carbonyl products.

The data suggest that the extremely strong induction of apoptosis and synergistic cytotoxicity of the anticancer drug (everolimus or barasertib) plus vitamin $\mathrm{C}$ and $\mathrm{K} 3$ does not require a strong production of ROS, in particularly hydroperoxides. However, the enhancement of proteincarbonyl products indicates an induction of oxidative stress. 
Presumably, other types of ROS (e.g. hydroxyl radicals produced in Fenton reactions) are involved in the synergistic cytotoxic effects of these triple combinations.

Other molecular mechanisms may also underlie these effects. For example, Chen et al. have shown that vitamin C and $\mathrm{K} 3$ reduce the activities of matrix metalloproteinases 2 and 9 , and urokinase plasminogen activator and thus inhibit tumor growth and exhibit anti-mestatatic potential in Lewis lung carcinoma (31). Tremante et al. have reported that subapoptotic doses of vitamin $\mathrm{C}$ and $\mathrm{K} 3$ sensitize human melanoma cells to natural killer cell lysis (32). This study suggests that reducing the dose of both vitamins could improve their ability to sensitize to everolimus and barasertib - abolishing cytotoxicity against normal lymphocytes and increasing the specificity of sensitization of leukemia lymphocytes. However, this assumption needs further verification.

Clearly, Jurkat leukemia lymphocytes are more sensitive to combined administration of anticancer drug (everolimus or barasertib) plus vitamin $\mathrm{C}$ and $\mathrm{K} 3$ as compared to normal cells. The combination of vitamin $\mathrm{C}$ plus $\mathrm{K} 3$ seems to be a powerful redox system that could specifically influence redox homeostasis of cancer cells and sensitize them to conventional chemotherapy.

\section{Acknowledgements}

This study was partially supported by the Center of Innovation Program from the Japan Science and Technology Agency and by the Diversity Grant 2017, NIRS-Chiba University, Japan (granted to R.B.).

\section{References}

1 Angsutararux $\mathrm{P}$, Luanpitpong $\mathrm{S}$ and Issaragrisil $\mathrm{S}$ : Chemotherapy-induced cardiotoxicity: Overview of the role of oxidative stress. Oxid Med Cell Longev 2015: 795602, 2015.

2 Jaiman S, Sharma AK, Singh K and Khanna D: Signaling mechanisms involved in renal pathological changes during cisplatin-induced nephropathy. Eur J Clin Pharmacol 69: 1863$1874,2013$.

3 Satoh M, Kashihara N, Fujimoto S, Horike H, Tokura T, Namikoshi T, Sasaki T and Makino H: A novel free radical scavenger, edarabone, protects against cisplatin-induced acute rental damage in vitro and in vivo. J Pharmacol Exp Ther 305 : 1183-1190, 2003

4 Ozben T: Oxidative stress and apoptosis: impact on cancer therapy. J Pharm Sci 96: 2181-2196, 2007.

5 Danz EDB, Skramsted J, Henry N, Bennett JA and Keller RS: Resveratrol prevents doxorubicin cardiotoxicity through mitochondrial stabilization and Sirt1 pathway. Free Radic Biol Med 46: 1589-1597, 2009.

6 Pathak AK, Bhutani M, Guleria R, Bail S, Mohan A, Mohanti BK, Sharma A, Pathak R, Bhardwaj NK, Prasad KN and Kochupillai V: Chemotherapy alone vs. chemotherapy plus highdose multiple antioxidants in patients with advanced non small cell lung cancer. J Am Coll Nutr 24: 16-21, 2005.
7 Lawenda BD, Kelly KM, Ladas EJ, Saga SM, Vickers A and Blumberg JB: Should supplement antioxidant administration be avoided during chemotherapy and radiation therapy? J Natl Cancer Inst 100: 773-783, 2008.

8 De Larco JE, Park CA, Dronava H and Furcht LT: Paradoxical roles for antioxidants in tumor prevention and eradication. Cancer Biol Ther 9: 362-370, 2010.

9 Zhelev Z, Ivanova D, Aoki I, Saga T and Bakalova R: 2-DeoxyD-glucose sensitizes cancer cells to barasertib and everolimus by ROS-independent mechanism(s). Anticancer Res 35: 6623$6632,2015$.

10 Zhelev Z, Ivanova D, Bakalova R, Aoki I and Higashi T: Induction of pentose-phosphate pathway through 6amininicitinamide sensitizes leukemia lymphocytes, but not normal lymphocytes to chemotherapeutics by ROS-independent mechanism. Anticancer Res 36: 6011-6020, 2016.

11 Zhelev Z, Ivanova D, Lazarova D, Aoki I, Bakalova R and Saga $\mathrm{T}$ : Docosahexaenoic acid sensitizes leukemia lymphocytes to barasertib and everolimus by ROS-dependent mechanisms without affecting the level of ROS and viability of normal lymphocytes. Anticancer Res 36: 1673-1682, 2016.

12 Zhelev Z, Ivanova D, Bakalova R, Aoki I and Higashi T: Synergistic cytotoxicity of melatonin and new-generation anticancer drugs against leukemia lymphocytes but not normal lymphocytes. Anticancer Res 37: 149-159, 2017.

13 McGuire K, Jamison J, Gilloteaux J and Summers JL: Vitamin C and $\mathrm{K} 3$ combination causes enhanced anticancer activity against RT-4 bladder cancer cells. J Cancer Sci Ther 5: 325-333, 2013.

14 Bonilla-Porras AR, Jimenez-Del-Rio M and Velez-Pardo C: Vitamin K3 and vitamin $\mathrm{C}$ alone or in combination induced apoptosis in leukemia cells by a similar oxidative stress signaling mechanism. Cancer Cell Int 11: 19, 2011.

15 Verrax J, Cadrobbi J, Marques C, Taper H, Habraken Y, Piette J and Calderon PB: Ascorbate potentiates the cytotoxicity of menadione leading to an oxidative stress that kills cancer cells by a non-apoptotic caspase-3 independent form of cell death. Apoptosis 9: 223-233, 2014.

16 Calderon P, Cadrobbi J, Marques C, Hong-Ngoc N, Jamison JM, Gilloteaux J, Summers JL and Taper HS: Potential therapeutic application of the association of vitamins $\mathrm{C}$ and $\mathrm{K} 3$ in cancer treatment. Curr Med Chem 9: 2271-2285, 2002.

17 Zhang W, Negoro T, Satoh K, Jiang Y, Hashimoto K, Kikuchi H, Nishikawa H, Miyata T, Yamamoto Y, Nakano K, Yasumoto E, Nakayachi $\mathrm{T}$, Mineno $\mathrm{K}$, Satoh $\mathrm{T}$ and Sakagami $\mathrm{H}$ : Synergistic cytotoxic action of vitamin $\mathrm{C}$ and vitamin K3. Anticancer Res 21: 3439-3444, 2001.

18 Ivanova D, Zhelev Z, Aoki I, Bakalova R and Higashi T: Overproduction of reactive oxygen species - obligatory or not for induction of apoptosis by anticancer drugs. Chin J Cancer Res 28: 53-66, 2016.

19 Witzig TE, Reeder C, Han JJ, LaPlant B, Stenson M, Tun HW, Macon W, Ansell SM, Habermann TM, Inwards DJ, Micallef IN, Johnston PB, Porrata LF, Colgan JP, Markovic S, Nowakowski GS and Gupta M: The mTORC1 inhibitor everolimus has antitumor activity in vitro and produces tumor responses in patients with relapsed T-cell lymphoma. Blood 126: 328-335, 2015.

20 Dengler J, von Bubnoff N, Decker T, Peschel C and Duyster J: Combination of imatinib with rapamycin or RAD001 acts synergistically only in BCR-ABL-positive cells with moderate resistance to imatinib. Leukemia 19: 1835-1838, 2015. 
21 Harituninians T, Mori A, O’Kelly J, Luong QT, Giles FJ and Koeffler HP: Antiproliferative activity of RAD001 (everolimus) as a single agent and combined with other agents in mantle cell lymphoma. Leukemia 21: 333-339, 2007.

22 Daver N, Boumber Y, Kantarjian H, Ravandi F, Cortes J, Rytting ME, Kawedia JD, Basnett J, Culotta KS, Zeng Z, Lu H, Richie MA, Garris R, Xiao L, Liu W, Baggerly KA, Jabbour E, O’Brien S, Burger J, Bendall LJ, Thomas D and Konopleva M: A Phase I/II study of the mTOR inhibitor everolimus in combination with hyperCVAD chemotherapy in patients with relapsed/refractory acute lymphoblastic leukemia. Clin Cancer Res 21: 2704-2714, 2015.

23 Mortlock AA, Foote KM, Heron NM, Jung FH, Pasquet G, Lohmann JJ, Warin N, Renaud F, De Savi C, Roberts NJ, Johnson T, Dousson CB, Hill GB, Perkins D, Hatter G, Wilkinson RW, Wedge SR, Heaton SP, Odedra R, Keen NJ, Crafter C, Brown E, Thompson K, Brightwell S, Khatri L, Brady MC, Kearney S, McKillop D, Rhead S, Parry T and Green S: Discovery, synthesis, and in vivo activity of a new class of pyrazoloquinazolines as selective inhibitors of aurora B kinase. J Med Chem 50: 2213-2224, 2007.

24 Porcelli L, Guida G, Quatrale AE, Cocco T, Sidella L, Maida I, Iacobazzi RM, Ferretta A, Stolfa DA, Strippoli S, Guida S, Tommasi S, Guida M and Azzariti A: Aurora kinase B inhibition reduces the proliferation of metastatic melanoma cells and enhances the response to chemotherapy. J Transl Med 13: 26, 2015.

25 Schwarz JBK, Langwieser N, Langwieser NN, Bek MJ, Seidl S, Eckstein H-H., Lu B, Schomig A, Pavenstadt H and Zohlnhofer D: Novel role of the CXC chemokine receptor 3 in inflammatory response to arterial injury: involvement of mTORC1. Circ Res 104: 189-200, 2009.

26 Pignochino Y, Dell'Aglio C, Basiricò M, Capozzi F, Soster M, Marchiò S, Bruno S, Gammaitoni L, Sangiolo D, Torchiaro E, D'Ambrosio L, Fagioli F, Ferrari S, Alberghini M, Picci P, Aglietta M and Grignani G: The combination of sorafenib and everolimus shows antitumor activity in preclinical models of malignant pleural mesothelioma. BMC Cancer 15: 374, 2015.
27 Pignochino Y, Dell'Aglio C, Basiricò M, Capozzi F, Soster M, Marchiò S, Bruno S, Gammaitoni L, Sangiolo D, Torchiaro E, D’Ambrosio L, Fagioli F, Ferrari S, Alberghini M, Picci P, Aglietta $\mathrm{M}$ and Grignani G: The combination of sorafenib and everolimus abrogates mTORC1 and mTORC2 up-regulation in osteosarcoma preclinical models. Clin Cancer Res 19: 21172131, 2013.

28 Zekri A, Mesbahi Y, Ghanizadeh-Vesali S, Alimoghaddam K, Ghavamzadeh A and Ghaffari SH: Reactive oxygen species generation and increase in mitochondrial copy number: new insight into the potential mechanism of cytotoxicity induced by aurora kinase inhibitor, AZD1152-HQPA. Anticancer Drugs 28: 841-851, 2017.

29 Kezic A, Thaiss F, Becker JU, Tsui TY and Bajcetic M: Effects of everolimus on oxidative stress in kidney model of ischemia/reperfusion injury. Am J Nephrol 37: 291-301, 2013.

30 Dando I, Pacchiana R, Pozza ED, Cataldo I, Bruno S, Conti P, Cordani M, Grimaldi A, Butera G, Caraglia M, Scarpa A, Palmieri $\mathrm{M}$ and Donadelli $\mathrm{M}$ : UCP2 inhibition induces ROS/AKT/mTOR axis: Role of GAPDH nuclear translocation in genipin/everolimus anticancer synergism. Free Radic Biol Med 113: 176-189, 2017.

31 Chen MF, Yang CM, Su CM, Liao JW and Hu ML: Inhibitory effect of vitamin $\mathrm{C}$ in combination with vitamin $\mathrm{K} 3$ on tumor growth and metastasis of Lewis lung carcinoma xenografted in C57BL/6 mice. Nutr Cancer 63: 1036-1043, 2011.

32 Tremante E, Santarelli L. Lo Monaco E, Sampaoli C, Ingegnere T, Guerrieri R, Tomasetti M and Giacomini P: Sub-apoptotic dosages of pr-oxidant vitamin cocktails sensitize human melanoma cells to NK cell lysis. Oncotarget 6: 31039-31049, 2015.

Received December 7, 2017

Revised January 16, 2018

Accepted January 24, 2018 